Rev Soc Esp Dolor

2015; 22(2): 89-90

\section{Objeciones que invalidan las conclusiones del estudio de efectividad, tolerabilidad y calidad de vida de tapentadol LP vs. oxicodona/naloxona LP}

\section{Sr. Director:}

"Razonar y convencer. ¿Qué difícil, largo y trabajoso! ¿Sugestionar? ¿Qué fácil, rápido y barato”. Sabias palabras del Dr. Ramón y Cajal. Las comunicaciones del estudio de tapentadol LP (TAP) vs. oxicodona/naloxona LP $(\mathrm{OXN})$ presentan un ensayo que parece diseñado para conseguir unos resultados que sugieran una superioridad de un fármaco sobre otro, no para comparar su eficacia y seguridad.

Es innegable el interés de un ensayo comparativo entre OXN y TAP. Ambos productos disponen de estudios pivotales doble ciego, de alto rigor metodológico, pero no se puede decir lo mismo del estudio cuyos resultados han sido presentados en los congresos SED 2014 (Simposio), WIP 2014 y PAINWeek 2014 (1-5) que concluyen que el TAP LP es la opción preferida para el tratamiento del dolor severo crónico con componente neuropático. Tan contundente afirmación basada en un estudio relativamente pequeño y abierto, y con diseño, conducción y análisis que implican fuertes sesgos a favor del TAP, parece totalmente arbitraria e injustificada, no avalada por los datos que presentan y el modo en que fueron obtenidos. A continuación se detallan varios puntos que ponen de manifiesto notables deficiencias y sesgos:

1. Estudio abierto vs. ciego, sesgos. Desde mediados del siglo pasado es universalmente aceptado, por la comunidad científica, que el riesgo de sesgo (consciente o no, intencionado o no) en los estudios abiertos es dramáticamente mayor que en los estudios doble ciegos (principalmente en medidas subjetivas como el dolor). La abundante bibliografía sobre el tema (p. ej. Day SJ, Altman DG. BMJ 2000; 321:504), no permite lugar a dudas al respecto.

2. Diseño y flujo de pacientes: brazo de rescate de TAP (pick-up arm), sesgos. Sesgo directamente relacionado con utilizar argumentos "que inducen a error" a investigadores y pacientes, al hacerles pensar que existen datos para afirmar que TAP ofrece mayores beneficios que OXN cuando este es el primer ensayo que compara ambos fármacos. El estudio determina que los pacientes tratados con OXN que no se controlen pueden pasar al brazo de rescate (pick-up arm) para ser tratados con TAP. Sin embargo, los que fracasan con TAP no se benefician de OXN y abandonan el estudio. La puerta al efecto nocebo (sugerir un efecto negativo asociado a un tratamiento y esta expectativa negativa ocurre), queda abierta en este estudio. ¿En qué se basan para describir los mayores beneficios de TAP sobre OXN?: a) mejor perfil de tolerabilidad de TAP $v s$. oxicodona mostrado en ensayos de fase III. No son ensayos vs. OXN; oxicodona y OXN tienen perfiles de tolerabilidad diferentes, como indican sus fichas técnicas; b) TAP tiene mejor tolerabilidad tras la rotación de opioides de tercer escalón de la OMS, argumentado con un estudio abierto en el que el grupo con el que se compara TAP proviene de "la combinación" de pacientes tratados con varios fármacos, y sólo 2 con $\mathrm{OXN}$. El atribuir a $\mathrm{OXN}$ los resultados del grupo de fármacos en el que se incluye es una práctica lamentable y esencialmente incorrecta; c) mejor perfil de efectividad y de impacto sobre los síntomas neuropáticos de TAP respecto otros opioides de tercer escalón. Basado en el mismo estudio que en el apartado anterior. Sobran comentarios adicionales; y d) superior perfil de TAP vs. OXN en un pool análisis pendiente de publicar en el momento de la aprobación del estudio. Objeción explícitamente clara ya que presenta como argumento resultados no publicados que además no son versus $\mathrm{OXN}$.

3. Diseño y manejo de cada fármaco, sesgos.

- Dosis y tiempos para la titulación. Se utilizan las pautas del empleo habitual de TAP en los dos grupos de tratamiento, ignorando las recomendaciones de la ficha técnica de OXN: no se permite utilizar la dosis de OXN 5/2,5 mg para el ajuste de dosis, se indican incrementos de $20 / 10 \mathrm{mg}$ cada subida de dosis en $24 \mathrm{~h}$ y los tiempos para poder subir la dosis, mínimo cada 3 días, están adaptados a las necesidades farmacocinéticas de TAP. Esto puede haber motivado el uso de dosis mayores de TAP $v s$. OXN en la titulación (20\% mayor a favor de TAP, considerando equianalgesia la proporción 5:1 TAP: OXN).

- Respuesta al tratamiento con disminución de un solo punto vs. el valor basal considerada suficiente en la fase de titulación cuando se han alcanzado las dosis máximas con cada fármaco. Lo habitual en los ensayos clínicos es considerar una reducción de dos puntos vs. la medida basal. A este criterio de baja exigencia, se suma el que se considere que es criterio suficiente, el común acuerdo entre médico y paciente, sin considerar escala alguna, respecto al 
alivio y la tolerabilidad para alcanzar la titulación y pasar al desarrollo del estudio. Esto podría favorecer el cambio de pacientes de OXN a TAP, o conservar en el brazo TAP a determinados pacientes.

4. Criterios de selección de los pacientes. Nä̈ve a opioides, no naiive a coanalgésicos, sesgo. Los criterios de selección obligan a que los pacientes sean nä̈ve a opioides (mecanismo principal de acción de $\mathrm{OXN}$ ), pero no se exige que sean naïve a coanalgésicos (con posibles principios de actuación similares a TAP), por lo que en realidad no serían "naïve" a TAP desde el punto de vista de que es un importante mecanismo de acción del fármaco.

5. Función intestinal. Otro objetivo del estudio que se está analizando es valorar la función intestinal y argumentan que no hay diferencia entre los beneficios que pueden aportar TAP y OXN a pesar de que precisamente OXN ofrece beneficios demostrados sobre el estreñimiento inducido por opioides, en estudios en los que se utilizan escalas validadas para medir esta variable. El estudio no demuestra superioridad de TAP vs. OXN utilizando la escala validada elegida en el estudio (PAC-SYM) y este era un objetivo primario del estudio.

Resumiendo, este es un estudio que muestra un grupo excepcionalmente elevado de procedimientos de diseño, ejecución y análisis, no recomendables en una investigación clínica de excelencia, que inducen a una conclusión no probada.

Conflicto de intereses: trabajo de revisión, análisis y crítica realizado a solicitud de Mundipharma España.

\section{A.J. Casado Collado ${ }^{1}$ y L. Prieto Valiente ${ }^{2}$}

${ }^{1}$ Departamento de Medicina Preventiva y Salud Pública. Facultad de Medicina UAM. Madrid
${ }^{2}$ Facultad de Medicina. Universidad Complutense de Madrid. Madrid

\section{BIBLIOGRAFÍA}

1. Baron R, Schwittay A, Binder A, Höper J, Helfert S, Falke $\mathrm{D}$, et al. Effectiveness of tapentadol prolonged release (PR) versus oxicodone/ naloxone PR for severe chronic low back pain with a neuropathic pain component. 7th World Congress World Institute of Pain Maastricht The Netherlands, 2014. Abstract WLP.0118.\&poster Abstract book pg 59.

2. Binder A, Baron R, Schwittay A, Höper J, Helfert S, Falke $\mathrm{D}$, et al. Safety/tolerability of tapentadol prolonged released (PR) versus oxycodone/naloxone PR for severe chronic low back pain with a neuropathic pain component. 7th World Congress World Institute of Pain Maastricht The Netherlands 2014. Abstract WLP-0229\&poster. Abstract book pg. 61.

3. Baron R, Schwittay A, Binder A, Schumann C, Falke D, Steigerwald I. Effectiveness of tapentadol prolonged release (PR) versus oxycodone/naloxone PR for severe chronic low back pain with a neuropathic pain component. PAIN Week, Las Vegas, USA 2014. Abstract 121. Available at: http://conference.painweek.org/media/mediafile_attachments/04/724painweek2014acceptedabstracts.pdf.

4. Binder A, Baron R, Schwittay A, Schumann C, Falke D, Steigerwald I. Safety and tolerability of tapentadol prolonged release (PR) versus oxycodone/naloxone PR for severe chronic low back pain with a neuropathic pain component. PAIN Week Las Vegas, USA 2014. Abstract 122. Available at: http://conference.painweek.org/media/ mediafile_attachments/04/724-painweek2014acceptedabstracts.pdf

5. Schwittay A, Baron R, Binder A, Schumann C, Falke D, Steigerwald I. Effects of tapentadol prolonged release (PR) versus oxycodone/naloxone PR on quality of life and function measures in patients with severe chronic low back pain with a neuropathic pain component. PAIN Week. Las Vegas, USA 2014. Abstract 123. Available at: http://conference.painweek.org/media/mediafile_attachments/04/724painweek2014acceptedabstracts.pdf 